\title{
Better efficacy of sequential combination with balloon pulmonary angioplasty after long-term riociguat for patients with inoperable chronic thromboembolic pulmonary hypertension
}

\section{Wei Wang}

Beijing Chao-Yang Hospital Capital Medical University: Beijing Chaoyang Hospital

Qiang Huang

Beijing Chao-Yang Hospital Capital Medical University: Beijing Chaoyang Hospital

\section{Suqiao Yang}

Beijing Chao-Yang Hospital Capital Medical University: Beijing Chaoyang Hospital

\section{Tuguang Kuang}

Beijing Chao-Yang Hospital Capital Medical University: Beijing Chaoyang Hospital Jianfeng Wang

Beijing Chao-Yang Hospital Capital Medical University: Beijing Chaoyang Hospital

\section{Yidan Li}

Beijing Chao-Yang Hospital Capital Medical University: Beijing Chaoyang Hospital Juanni Gong

Beijing Chao-Yang Hospital Capital Medical University: Beijing Chaoyang Hospital

Yuanhua Yang ( $\nabla$ yyh1031@sina.com )

Beijing Chao-Yang Hospital https://orcid.org/0000-0003-0293-6737

Research article

Keywords: balloon pulmonary angioplasty, chronic thromboembolic pulmonary hypertension, echocardiography, pulmonary hypertension, riociguat

Posted Date: February 23rd, 2022

DOI: https://doi.org/10.21203/rs.3.rs-1334570/v1

License: (9) (1) This work is licensed under a Creative Commons Attribution 4.0 International License. Read Full License 


\section{Abstract}

Background: There is little data showing further benefit for the chronic thromboembolic pulmonary hypertension (CTEPH) patients who take long-term riociguat undergoing balloon pulmonary angioplasty (BPA) procedures. The present study aimed to evaluate the efficacy of long-term riociguat sequentially combined with BPA for patients with inoperable CTEPH.

Methods: Eight inoperable CTEPH patients were enrolled in this study, who have been administrated riociguat $2.5 \mathrm{mg}$ three times daily for about eight years, then underwent several sessions of BPA procedures. Data are prospectively collected to evaluate clinical outcomes, hemodynamics, exercise capacity, and right heart size and function by echocardiography at baseline, eight years after riociguat, and three months after the final BPA.

Results: Eight patients (mean age $54.9 \pm 11.4$ years) had accepted riociguat $2.5 \mathrm{mg}$ three times daily for $95.0 \pm 10.7$ months. Cardiac index $(\mathrm{Cl})\left(1.5 \pm 0.5 \mathrm{~L} / \mathrm{min} / \mathrm{m}^{2}\right.$ to $\left.2.4 \pm 0.6 \mathrm{~L} / \mathrm{min} / \mathrm{m}^{2}, \mathrm{P}=0.005\right), 6$ minutes walking distance (6MWD) (329.6 $\pm 87.5 \mathrm{~m}$ to $418.1 \pm 75.8 \mathrm{~m}, \mathrm{P}=0.016)$, and pulmonary vascular resistance (PVR) (1336.9 \pm 320.2 dyn $\cdot \mathrm{s} \cdot \mathrm{cm}^{-5}$ to $\left.815.4 \pm 195.6 \mathrm{dyn} \cdot \mathrm{s} \cdot \mathrm{cm}^{-5}, \mathrm{P}=0.008\right)$ were significant improvement after riociguat treatment. A total of 33 additional combinational BPA sessions (mean 4.1 \pm 1.6 ) and 150 balloon dilations (mean 18.8 \pm 8.1 ) were performed. Mean pulmonary artery pressure (mPAP) $(54.1 \pm 11.1 \mathrm{mmHg}$ to $33.6 \pm 7.7 \mathrm{mmHg}, \mathrm{P}=0.002)$ and PVR $\left(815.4 \pm 195.6 \mathrm{dyn} \cdot \mathrm{s} \cdot \mathrm{cm}^{-5}\right.$ to $\left.428.3 \pm 151.2 \mathrm{dyn} \cdot \mathrm{s} \cdot \mathrm{cm}^{-5}, \mathrm{P} 囚 0.001\right)$ were further decreased. $\mathrm{Cl}\left(2.4 \pm 0.6 \mathrm{~L} / \mathrm{min} / \mathrm{m}^{2}\right.$ to $\left.2.7 \pm 0.7 \mathrm{~L} / \mathrm{min} / \mathrm{m}^{2}\right)$ and $6 \mathrm{MWD}(418.1 \pm 75.8 \mathrm{~m}$ to $455.7 \pm 100.0 \mathrm{~m}$ ) were increased significantly.

Conclusions: After long-term riociguat treatment, sequential combination with BPA delivered considerably incremental benefits on exercise capacity and pulmonary hemodynamics, as well as right heart size and function of technically inoperable CTEPH patients.

Trial registration: The study is registered on ClinicalTrials.gov in the 27 March 2020 (NCT04326777).

\section{Introduction}

Chronic thromboembolic pulmonary hypertension (CTEPH), as the fourth group of pulmonary hypertension $(\mathrm{PH})$, is characterized by organized thrombi, pulmonary arteries remodeling resulting in stenosis or occlusion, progressive pulmonary pressure, and finally leading to right heart failure, and carries a poor prognosis[1]. According to the 2015 ERS/ESC Pulmonary Hypertension Guidelines, PEA is a potentially curative surgical treatment option for the majority of patients, and has become the preferred therapeutic strategy for CTEPH[2]. However, PEA is only suitable for those patients whose lesions mainly located in the main, lobar and segmental pulmonary arteries[3]. Approximately $40 \%$ of CTEPH patients with peripheral pulmonary arteries obstruction and those with comorbidities have been deemed unfavorable risk/benefit ratio are not amenable to the PEA. Moreover, studies have found that there is up to $35 \%$ residual pulmonary hypertension after PEA[4]. 
Microvascular arteriopathy in CTEPH provides a theoretical basis for the application of PH-targeted medications. Several clinical trials have justified the efficacy of PH-targeted medical therapy in CTEPH with patients who are technically inoperable or postoperative persistent pulmonary hypertension or have an unacceptable risk/benefit ratio for PEA[5-8]. Riociguat, a stimulator of soluble guanylate cyclase, which was shown to significantly improve patient's exercise capacity, pulmonary vascular resistance (PVR), the N-terminal fragment of pro-brain natriuretic peptide (NT-proBNP) levels, and had an estimated 1-year survival of $97 \%$ in the CHEST trails, is the first drug approved for inoperable CTEPH or persistent/recurrent CTEPH after PEA[9, 10].

Balloon pulmonary angioplasty (BPA), also known as percutaneous transluminal pulmonary angioplasty (PTPA), is a currently established alternative therapy for inoperable patients with CTEPH. It was first covered in a case report in 1988, which showed positive results in mean pulmonary artery pressure(mPAP) and pulmonary perfusion scan[11]. It wasn't until 2012 that BPA was shown to safety and efficacy with a refined approach using smaller size balloon for fewer lobes per procedure for nonoperable CTEPH patients[12]. With growing worldwide experience with BPA, its role in CTEPH treatment have evolved at a rapid pace, and will be updated in the next guidelines as the intervention[13]. The 2015 European Guideline recommends that riociguat or BPA is considered in patients who have been classified as persistent/recurrent CTEPH after PEA or who are technically non-operable or carry an unfavourable risk:benefits ratio for $\mathrm{PEA}$ [2]. The use of targeted-PH medicine to decrease pulmonary hypertension prior to BPA procedure is common. However, there is little data to show further benefit for the CTEPH patients who take long-term riociguat undergoing BPA procedures.

Therefore, the present study aimed to evaluate the efficacy of long-term riociguat and sequential combination with BPA procedure for the inoperable CTEPH patients, and to discover whether BPA had an additional benefit for CTEPH patients who were administrated long-term riociguat. Our pulmonary hypertension center has been participating in the multi-center international CHEST study, several participants have been taking riociguat for about eight years, therefore, which creating conditions for the implementation of the study.

\section{Methods}

\section{Patient selection}

This is a single-center, observational study. Eight patients, who participated in the international multicenter CHEST study from September 2009 to May 2020 in our PH center, were enrolled in the study. All these patients, who were technically inoperable $\mathrm{CTEPH}$, have been receiving long-term riociguat of $2.5 \mathrm{mg}$ three times daily for almost eight years, then sequentially combined with at least 2 BPA procedures at our center. The study was approved by the ethics committee of Beijing Chaoyang Hospital, Capital Medical University (NO.2019-KE-377), complies with the Declaration of Helsinki. All the patients provided their written informed consents. The study is registered on ClinicalTrials.gov (NCT04326777).

\section{Data Collection}


Data were collected at three time points: before riociguat (baseline), before the first BPA, and at least three months after the final BPA, including medical history, age, gender, body mass index (BMI), comorbidities, NT-proBNP, WHO FC, 6MWD, hemodynamic parameters measured by Swan-Ganz right heart catheterization and echocardiographic parameters. In terms of BPA, the frequency of BPA procedures, the number of dilated vessels and complications were documented.

\section{Targeted medical therapy}

Patients enrolled in the international CHEST study, were administrated riociguat adjusted from a starting dose of $1 \mathrm{mg}$ three times daily up to a maximum of $2.5 \mathrm{mg}$ three times daily before BPA depending on the patient's systolic blood pressure (SBP), side-effects and progressive CTEPH. The dose was not changed throughout the study. Those patients didn't accept oral other PH medications combination therapy.

\section{Balloon pulmonary angioplasty}

BPA was step-wise performed by two skilled interventional radiologists. A conventional femoral venipuncture was made. A 6F JR- or JL-guided catheter (Launcher, Cordis, Miami Lakes, FL, USA) was introduced to the target vessel by a guidewire. A 2-mm balloon was used for the first vascular expansion. Then the balloon was gradually inflated to a size no larger than the target vessel. In case of complications during the BPA procedure, the treatment was immediately made, including seal of the injury using a balloon catheter, bioabsorbable gelatin or metallic coil and the insertion of covered stents. Patients were discharged at least one day after each BPA session to observe refusion pulmonary edema and contrast kidney injury. Warfarin was routinely used at time of diagnosis of CTEPH and during all courses, the international normalized ratio was maintained between 2.0 and 3.0. Diuretics and vasoactive agents were given according to preoperative right heart function. For patients with estimated Glomerular Filtration Rate $(\mathrm{eGFR})<60 \mathrm{~mL} /\left(\mathrm{min}^{\star} 1.73 \mathrm{~m}^{2}\right), 0.9 \%$ sodium chloride injection was provided to hydrate at the rate of $0.5-1 \mathrm{ml} / \mathrm{kg} / \mathrm{h}$ before BPA procedure to avoid the occurrence of postoperative acute contrast kidney injury. Most patients need several series of BPA procedures and each series of procedure includes two sessions. The odd-numbered intervals between the BPA sessions were no less than one month, and the even-numbered intervals were no less than two weeks. The number of BPA sessions, the total number of dilated vessels (sub-segmental level), and number of dilated vessels per session were collected. The treatment endpoint of this study is no less than $20 \%$ decrease in PVR level comparing with that before BPA procedures.

\section{Statistical analysis}

Continuous variables are expressed as mean $\pm \mathrm{SD}$ or as median and interquartile range (IQR). Categorical variables are documented as numbers and percentages. Friedman test is used for variables not conforming to normal distribution or categorical variables. The data measured at the different time points are compared using one-way repeated measure analysis of variance. All statistical tests were performed with SPSS software version 24.0 (SPSS Inc; Chicago, Illinois, USA). All charts were made using GraphPad Prism 8.0 (GraphPad Software, La Jolla, CA, USA). P-value $<0.05$ was considered to be statistically significant. 


\section{Results}

\section{Patient characteristics}

All the eight patients are technically inoperable CTEPH, the characteristics of the patients' baseline data are demonstrated in Table 1. From September 2009 to May 2020, 8 patients (mean age 54.9 \pm 11.4 years,7 women, $1 \mathrm{man}$ ) who enrolled in CHEST-1 and CHEST-2 have been administrated riociguat $2.5 \mathrm{mg}$ three times daily for $95.0 \pm 10.7$ months. The period from the first symptoms to diagnosis of CTEPH was 15.0 months (Q1-Q3:12.0-36.0 months, $\mathrm{n}=8$ ). All patients have been giving warfarin to maintain anticoagulant therapy. During the CHEST-2 study, sequential combination with BPA procedures were implemented. The distribution of chronic thromboembolic materials did not change at baseline and pre-BPA by pulmonary angiography after effective anticoagulants.

Table 1. Patients' Characteristics at Baseline

\begin{tabular}{|ll|}
\hline Variables & Baseline \\
\hline Subjects $(\mathrm{n})$ & 8 \\
\hline Age (years) & $54.9 \pm 11.4$ \\
\hline Female, $\mathrm{n}(\%)$ & $7(87.5)$ \\
\hline BMI $\left(\mathrm{kg} / \mathrm{m}^{2}\right)$ & $25.9 \pm 2.6$ \\
\hline Previous VTE, $\mathrm{n}(\%)$ & $5(62.5)$ \\
\hline Period from symptoms to CTEPH diagnosis (months) & $15.0(12.0-36.0)$ \\
\hline Period of riociguat used (months) & $95.0 \pm 10.7$ \\
\hline Vitamin K antagonist & $8(100)$ \\
\hline Comorbidities, $\mathrm{n}$ (\%) & $5(62.5)$ \\
\hline Hypertension & $2(25.0)$ \\
\hline Hyperlipidemia & $1(12.5)$ \\
\hline Type 2 diabetes & $1(12.5)$ \\
\hline Gynecological disease & $1(12.5)$ \\
\hline Lower limb vascular disease & $1(12.5)$ \\
\hline
\end{tabular}

Data are expressed as mean \pm SD or number(percentage) or median with first and third quartiles (Q1:Q3). BMI: body mass index; BPA: balloon pulmonary angioplasty; CTEPH: chronic thromboembolic pulmonary hypertension; VTE: venous thrombus embolism.

The outcomes of targeted medicine and BPA procedure 
The exercise capacity, hemodynamics, serum NT-proBNP level at baseline, before BPA (riociguat treatment) and after final BPA (BPA treatment) are presented in Table 2 and Figure 1. There were improvements in 6WMD (329.6 $\pm 87.5 \mathrm{~m}$ to $455.7 \pm 100.0 \mathrm{~m}, \mathrm{P}=0.003)$, WHO FC (improvement in 7 patients) and NT-proBNP level (1650.0 $\pm 1136.3 \mathrm{pg} / \mathrm{mL}$ to $169.5(49.25-603.0) \mathrm{pg} / \mathrm{mL}, \mathrm{P}=0.003)$. The hemodynamics showed improvements in mPAP $(51.9 \pm 6.3 \mathrm{mmHg}$ to $33.6 \pm 7.7 \mathrm{mmHg}, \mathrm{P}=0.011), \mathrm{CO}(2.8 \pm 0.9 \mathrm{~L} / \mathrm{min}$ to 4.6 $\pm 1.3 \mathrm{~L} / \mathrm{min}, \mathrm{P}=0.008), \mathrm{Cl}\left(1.5 \pm 0.5 \mathrm{~L} / \mathrm{min} / \mathrm{m}^{2}\right.$ to $\left.2.7 \pm 0.7 \mathrm{~L} / \mathrm{min} / \mathrm{m}^{2}, \mathrm{P}=0.005\right), \mathrm{PVR}(1336.9 \pm 320.2$ dyn $\cdot s \cdot \mathrm{cm}^{-5}$ to $\left.428.3 \pm 51.2 \mathrm{dyn} \cdot \mathrm{s} \cdot \mathrm{cm}^{-5}, \mathrm{P}=0.001\right), \mathrm{SvO}_{2}(55.6 \pm 11.7 \%$ to $65.3 \pm 3.6 \%, \mathrm{P}=0.056)$. Certain parameters indicating right heart size and function by echocardiography were showed in Table 3 , comparation with these data at baseline were also improved. 
Table 2

Functional capacity, hemodynamics at baseline, before BPA and after BPA.

\begin{tabular}{|c|c|c|c|c|}
\hline Variables $(n=8)$ & Baseline & Before BPA & After BPA & $P$ \\
\hline \multicolumn{5}{|l|}{ Exercise capacity } \\
\hline \multicolumn{5}{|l|}{ WHO FC (n) } \\
\hline 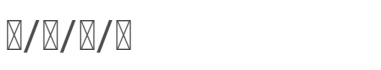 & $0 / 2 / 6 / 0$ & $3 / 4 / 1 / 0$ & $4 / 3 / 1 / 0$ & 0.761 \\
\hline 6MWD (m) & $329.6 \pm 87.5$ & $418.1 \pm 75.8^{*}$ & $455.7 \pm 100.0^{\dagger}$ & $<0.001$ \\
\hline NT-proBNP(pg/mL) & $1650.0 \pm 1136.3$ & $440.5(257.5-1522.75)$ & $169.5(49.25-603.0)$ & 0.005 \\
\hline \multicolumn{5}{|l|}{ Hemodynamics } \\
\hline RAP, mmHg & $10.0 \pm 5.5$ & $8.8 \pm 2.8$ & $6.9 \pm 2.3$ & 0.169 \\
\hline sPAP, mmHg & $92.6 \pm 11.4$ & $90.8 \pm 19.0$ & $54.3 \pm 12.6^{\dagger}$ & 0.001 \\
\hline $\mathrm{dPAP}, \mathrm{mmHg}$ & $31.8 \pm 5.2$ & $35.6 \pm 8.6$ & $22.9 \pm 5.3^{\dagger}$ & 0.001 \\
\hline mPAP, mmHg & $51.9 \pm 6.3$ & $54.1 \pm 11.1$ & $33.6 \pm 7.7^{\dagger}$ & 0.001 \\
\hline PAWP, mmHg & $8.8 \pm 2.0$ & $12.9 \pm 6.4$ & $9.7 \pm 2.1$ & 0.371 \\
\hline $\mathrm{CO}, \mathrm{L} / \mathrm{min}$ & $2.8 \pm 0.9$ & $4.1 \pm 1.1^{*}$ & $4.6 \pm 1.3$ & 0.001 \\
\hline $\mathrm{Cl}, \mathrm{L} / \mathrm{min} / \mathrm{m}^{2}$ & $1.5 \pm 0.5$ & $2.4 \pm 0.6^{*}$ & $2.7 \pm 0.7$ & $<0.001$ \\
\hline PVR, dyn $\cdot s \cdot \mathrm{cm}^{-5}$ & $1336.9 \pm 320.2$ & $815.4 \pm 195.6^{*}$ & $428.3 \pm 151.2^{\dagger}$ & $<0.001$ \\
\hline $\mathrm{SvO}_{2}, \%$ & $55.6 \pm 11.7$ & $55.4 \pm 8.2$ & $65.3 \pm 3.6^{\dagger}$ & 0.027 \\
\hline \multicolumn{5}{|c|}{$\begin{array}{l}\text { Data are expressed as mean } \pm \text { SD or number(percentage) or median with first and third quartiles } \\
\text { (Q1:Q3). 6MWD:6 minutes walking distance; BPA: balloon pulmonary angioplasty; Cl: cardiac index; } \\
\text { CO: cardiac output; dPAP: diastolic pulmonary arterial pressure; mPAP: mean pulmonary arterial } \\
\text { pressure; NT-proBNP: N-terminal fragment of pro-brain natriuretic peptide; PAWP: pulmonary arterial } \\
\text { wedge pressure; PVR: pulmonary vascular resistance; RAP: right atrial pressure; sPAP: systolic } \\
\text { pulmonary arterial pressure; } \mathrm{SvO}_{2} \text { : mixed venous oxygen saturation; WHO FC: world health }\end{array}$} \\
\hline
\end{tabular}


Table 3

Echocardiographic parameters at baseline, before BPA and after BPA.

\begin{tabular}{|lllll|}
\hline Echocardiographic data (n=8) & Baseline & Before BPA & After BPA & $P$ \\
\hline LVEDD, mm & $36.0(28.4-37.5)$ & $40.0 \pm 4.1$ & $46.3 \pm 3.2$ & $<0.001$ \\
\hline RV basal diameter, mm & $47.3 \pm 7.6$ & $49.0(46.0-51.0)$ & $41.3 \pm 5.5$ & 0.115 \\
\hline RV/LV diameter ratio & $1.6 \pm 0.5$ & $1.2 \pm 0.2$ & $1.1 \pm 0.1$ & 0.028 \\
\hline RV free wall thickness, mm & $6.4 \pm 1.3$ & $7.0 \pm 1.8$ & $6.3 \pm 0.9$ & 0.374 \\
\hline MPAD, mm & $30.0 \pm 4.5$ & $39.9 \pm 12.3$ & $37.8 \pm 12.6$ & 0.089 \\
\hline RA minor dimension, mm & $49.3 \pm 8.8$ & $52.0(48.8-53.3)$ & $46.6 \pm 8.9$ & 0.419 \\
\hline TRPD, mmHg & $84.8 \pm 10.5$ & $91.1 \pm 24.5$ & $56.2 \pm 16.5$ & 0.013 \\
\hline TAPSE, mm & NA & $16.7 \pm 3.0$ & $17.1 \pm 3.7$ & 0.500 \\
\hline $\begin{array}{l}\text { IVC diameter, mm } \\
\text { RV FAC, \% }\end{array}$ & NA & $24.3 \pm 6.0$ & $19.0 \pm 4.8$ & 0.040 \\
\hline $\begin{array}{l}\text { Data are expressed as mean } \pm \text { SD or number (percentage) or median with first and third quartiles } \\
\text { (Q1:Q3). IVC: inferior vena cava; LV: left ventricular; LVEDD: left ventricular end-diastolic diameter; }\end{array}$ \\
$\begin{array}{l}\text { MPAD: main pulmonary artery diameter; RA: right atrial; RV: right ventricular; RV FAC: right ventricular } \\
\text { fractional area change; TAPSE: tricuspid annular plane systolic excursion; TRPD: tricuspid } \\
\text { regurgitation pressure difference. }\end{array}$
\end{tabular}

The study illuminates that $\mathrm{PH}$-targeted medical therapy has positive benefits on CTEPH patients. There are increases in $6 \mathrm{MWD}$ of almost $100 \mathrm{~m}(329.6 \pm 87.5 \mathrm{~m}$ to $418.1 \pm 75.8 \mathrm{~m}, \mathrm{P}=0.016)$. WHO FC are improved by one class in 6 of 8 patients and two classes in 1 of 8 patients $(P=0.011)$. NT-pro BNP values are noted from $1650.0 \pm 1136.3 \mathrm{pg} / \mathrm{mL}$ to $440.5(257.5-1522.75) \mathrm{pg} / \mathrm{mL}$, but no statistical different. Hemodynamics assessment showed significant improvement in $\mathrm{CO}$ of $1.3 \mathrm{~L} / \mathrm{min}(2.8 \pm 0.9 \mathrm{~L} / \mathrm{min}$ to $4.1 \pm 1.1 \mathrm{~L} / \mathrm{min}$, $\mathrm{P}=0.015)$, and in $\mathrm{Cl}$ of $0.9 \mathrm{~L} / \mathrm{min} / \mathrm{m}^{2}\left(1.5 \pm 0.5 \mathrm{~L} / \mathrm{min} / \mathrm{m}^{2}\right.$ to $\left.2.4 \pm 0.6 \mathrm{~L} / \mathrm{min} / \mathrm{m}^{2}, \mathrm{P}=0.005\right)$. Meanwhile, riociguat administration makes the decline of PVR by $521.5 \mathrm{dyn} \cdot \mathrm{s} \cdot \mathrm{cm}^{-5}\left(1336.9 \pm 320.2 \mathrm{dyn} \cdot \mathrm{s} \cdot \mathrm{cm}^{-5}\right.$ to $815.4 \pm 195.6 \mathrm{dyn} \cdot \mathrm{s} \cdot \mathrm{cm}^{-5}, \mathrm{P}=0.008$ ). The CTEPH patients who received riociguat achieve not only significant improvements in $6 \mathrm{MWD} \square \mathrm{CO} \triangle \mathrm{Cl}$ but also a significant reduction in PVR. However, the pulmonary arterial pressure slightly elevated, and $\mathrm{SvO}_{2}$ remains almost similar. The echocardiographic indicators are demonstrated in Table3. There is only an improvement in LVEDD (36.0(28.4-37.5mm)to $40.0 \pm 4.1 \mathrm{~mm}, \mathrm{P}=0.24)$ and $\mathrm{RV} / \mathrm{LV}$ diameter $(1.6 \pm 0.5$ to $1.2 \pm 0.2, \mathrm{P}=0.23)$, while no statistical differences. Furthermore, other parameters show that right heart structure continue to deterioration.

After eight years riociguat administration, the eight patients were treated with a total of 33 sequential combination BPA sessions (mean $4.1 \pm 1.6$ ) and 150 balloon dilations (mean 18.8 \pm 8.1 ). Pulmonary arterial pressure indicators significantly decrease in SPAP $(90.8 \pm 19.0 \mathrm{mmHg}$ to $54.3 \pm 12.6 \mathrm{mmHg}$, $P=0.001)$, dPAP $(35.6 \pm 8.6 \mathrm{mmHg}$ to $22.9 \pm 5.3 \mathrm{mmHg}, P=0.003), \mathrm{mPAP}(54.1 \pm 11.1 \mathrm{mmHg}$ to $33.6 \pm 7.7$ 
$\mathrm{mmHg}, \mathrm{P}=0.002)$, respectively. Based on riociguat administration, $\mathrm{CO}$ and $\mathrm{Cl}$ are further increased from $4.1 \pm 1.1 \mathrm{~L} / \mathrm{min}$ to $4.6 \pm 1.3 \mathrm{~L} / \mathrm{min}, 2.4 \pm 0.6 \mathrm{~L} / \mathrm{min} / \mathrm{m}^{2}$ to $2.7 \pm 0.7 \mathrm{~L} / \mathrm{min} / \mathrm{m}^{2}$ after several BPA sessions, respectively. $\mathrm{SvO}_{2}$ also statistically improves from $55.4 \pm 8.2 \%$ to $65.3 \pm 3.6 \%(P=0.038)$. PVR declines further from $815.4 \pm 195.6 \mathrm{dyn} \cdot \mathrm{s} \cdot \mathrm{cm}^{-5}$ to $428.3 \pm 151.2 \mathrm{dyn} \cdot \mathrm{s} \cdot \mathrm{cm}^{-5}(\mathrm{P} \otimes 0.001)$. NT-proBNP are further lessened from $440.5(257.5-1522.75) \mathrm{pg} / \mathrm{mL}$ to $169.5(49.25-603.0) \mathrm{pg} / \mathrm{mL}$. In the aspect of exercise capacity, $6 \mathrm{MWD}$ is significantly improved from $418.1 \pm 75.8 \mathrm{~m}$ to $455.7 \pm 100.0 \mathrm{~m}$ ( $P=0.038)$. No statistically significant difference is noted in WHO FC, but improvement comparing with values before BPA

procedures. The echocardiographic parameters show that RV basal diameters, RV/LV basal diameter, RV free wall thickness, right atrial dimension, IVC diameter, TAPSE and RV FAC are improved to some extent, compared with indicators at baseline and after riociguat. Although those parameters show no statistical difference, it still shows a reduction in right heart size and an elevate in right heart function.

Representative echocardiograms are shown in Figure 2 and Figure 3.

\section{Complications of BPA}

There were 7 procedure related complications occurred during the 33 interventions ( $21.2 \%$ of all interventions). These adverse events were mostly caused by wire perforation of the pulmonary vasculature, with mild hemoptysis in 5 BPA sessions. There was asymptomatic, only observed a bit contrast effusion during 1 BPA session. 1 patient developed reperfusion edema with deteriorated dyspnea and desaturation during the post-procedural period of $24 \mathrm{~h}$. Non-invasive ventilation was used to oxygen therapy. No procedure-related death occurred.

\section{Discussion}

With the recognition of $\mathrm{CTEPH}$, the therapy strategies are updated gradually, including the preferred PEA, targeted medicine and emerging interventional treatment. According to the guidelines, riociguat is the first medication recommened for persistent/recurrent PH after PEA or inoperable CTEPH. In 2012, Janpanese investigators refined the BPA procedure to make it an alternative therapeutic approch for patients with inoperable CTEPH. However, it is not clear whether patients who receive long-term riociguat still benefit from BPA procedures. Our current study demonstrates the exercise tolerance and hemodynamics are further improved after long-term riociguat and sequential combination with BPA procdures for the inoperable CTEPH. The main findings of the present study are that(1)riociguat continues to improve exercise capacity and hemodynamics over the long term; (2)the combination of riociguat and sequential BPA procedures generates further improvement in CO,Cl, PVR ,6WMD and NT-proBNP, in addition to decreasing the mPAP; (3)BPA can reverse RV remodeling and improve RV function. These fingdings suggest that BPA can further increase the beneficial effects on inoperable CTEPH patients who take longterm riociguat.

In the CHEST-1 study, there were significant improvements in the $6 \mathrm{MWD}$ by $39 \pm 79 \mathrm{~m}$, the NT-proBNP level by $-291 \pm 1717 \mathrm{pg} / \mathrm{ml}$ and $\mathrm{WHO}$ function class by $33 \%$, the CO by $0.8 \pm 1.1 \mathrm{~L} / \mathrm{min}$, as well as a mean reduction in PVR of $226 \mathrm{dyn} \cdot \mathrm{s} \cdot \mathrm{cm}^{-5}$ [10]. In a 1-year open-label extension trial (CHEST-2), improvements in 
6MWD and WHO FC were maintained, with a survival rate of $97 \%$ and a rate of clinical worsening-free survival of $88 \%$ at 1 year[9]. Nevertheless, long-term outcome more than one year wasn't showed in the CHEST study. In our present cohort, exercise capacity and hemodynamics parameters have improved for up to eight years after riociguat treatment and further improvements after BPA. Based on the results of several previous studies, exercise capacity and hemodynamics were associated with prognosis and overall survival among patients with $\mathrm{CTEPH}[14,15]$. Hence, our findings clearly suggest the benefits of the sequential combinational treatment with riociguat and BPA.

However, right heart size and function assessed by echocardiography have no significant changes during long-term treatment with riociguat, and even tend to deteriorate. The present result contradicts previous data showing that patients under long-term treatment with riociguat demonstrated significantly reduced right heart size and improved RV function in PAH and CTEPH[16]. In the previous long-term riociguat study, the duration of administration was just 12 months, while our present study lasts 8 years whereby the results were beneficial, attesting to the long-term efficacy of riociguat. However, the study also shows an increased in mPAP, over-sized right heart and clinical worsening in some patients. As we know, CTEPH is a progressive condition. The CHEST study has shown that riociguat is not a curable treatment. Therefore, it is not surprising that patients who are technically inoperable or postoperative persistent pulmonary hypertension or have an unacceptable risk/benefit ratio for PEA, treated with riociguat, whose pulmonary hemodynamic/RV size and function may deteriorate over time. These findings suggest that riociguat alone is insufficient for the treatment in this group. The riociguat therapy can only improve the $\mathrm{CO}$ of right heart, nevertheless, the structure of right heart and function still gradually deteriorate due to the persistent pulmonary hypertension.

Since Japanese investigators refining the BPA procedure in 2012, it has rapidly spread around the world due to its dramatically improved efficacy and safety. Published data from different countries have shown significant improvements in hemodynamics, exercise capacity and markers of heart failure such as BNP or NT-proBNP level[17-19]. Currently, BPA carries a class $₫ b$ recommendation for the treatment of inoperable CTEPH in the European guidelines[2]. PH-targeted medical therapy is common in CTEPH patients prior to treatment with BPA procedure, however, the further benefits of this approach remain unclear. In a small randomized controlled study by Sugimura et al., patients with inoperable CTEPH were treated with medical therapy prior to BPA for 1-3 months, which resulted in improved $\mathrm{CO} / \mathrm{Cl}$, and PVR, but little change in mPAP and 6MWD[20], which demonstrated that BPA combined with conventional vasodilator treatment was quite effective in terms of improving the pulmonary hemodynamics and shortterm prognosis in patients with distal-type CTEPH. In another study of 36 patients by Wiedenroth et al, patients with inoperable CTEPH were given riociguat before BPA for mean five months, which suggested that the combination of riociguat and BPA was an effective treatment for patients with inoperable CTEPH, leading to significant improvements in physical capacity and pulmonary hemodynamics[21]. These findings illuminated that the short-term use of targeted-PH agents prior to BPA, which contributed to the improvements of right heart function and hemodynamics of the inoperable CTEPH patients, may guarantee the safety of BPA procedure by reducing the incidence of reperfusion pulmonary edema. Our present study showed that BPA generated further benefits for who take riociguat up to 8 years. Kaspar 
Broch et al. reported a significant improvement in RV functional parameters by echocardiography after BPA in 2015, which indicated credibility to this form of treatment in patients with CTEPH who undergo BPA[22]. In our current study, the changes of right heart size and function by echocardiography after BPA procedure are in line with the previous data. Thus, our study also supports the viewpoint that BPA procedure can reverse the remodeling of right heart structure and function.

Our results demonstrated that long-term riociguat and sequential combination with BPA is an effective treatment for patients with inoperable CTEPH. However, riociguat regulates the NO-sGC-cGMP pathway to prevent the development of $\mathrm{PH}$, the use of anticoagulants for inoperable CTEPH patients merely prevent new thrombosis and reduce pulmonary arteries block. Therefore, medical therapy has limited effect, even long-term medication cannot avoid the aggravation of the disease. BPA procedure improves CTEPH patients' hemodynamics and exercise tolerance through several mechanisms. Firstly, an overall vessel expansion induced by the stretching of the arterial wall leads to lumen enlargement immediately after BPA[23]. Secondly, the BPA relieves hemodynamic stress towards the non-BPA-side lung and subsequently decreases the non-BPA-side PVR in patients with CTEPH, which plays a significant role in suppression or possibly regression of small-vessel arteriopathy in these patients[24]. The last but not least, BPA procedure improves total pulmonary artery compliance in proportion to a decrease in PVR[25]. Therefore, the BPA procedure directly opens the mechanical obstruction vessels through the above mechanisms to reduce pulmonary vascular resistance and pulmonary artery pressure, thereby decreasing the right cardiac load, significantly reversing remodeling of the RV structure as well as the improvement of RV function, and further improving the prognosis of patients.

Some limitations to this study must be considered. First, this is a single-center observational study. In addition, the number of patients enrolled was small. Furthermore, we have no matched control group. However, our research has its advantages. Namely, we selected patients from the CHEST study in China and our results derived from clinical real observational data. Those patients enrolled in study have taken riociguat for almost eight years, but their mPAP, right heart size and function did not improve significantly, even some patients were clinically worse. Given this, we did sessions of BPA procedures for them, which leading to decreasing mPAP, further enhancement in $\mathrm{CO} / \mathrm{Cl}, \mathrm{PVR}$, especially in right heart size and function by echocardiography. We confirmed not only the long-term efficacy of riociguat in patients with inoperable CTEPH, but also further benefits from BPA procedure.

\section{Conclusion}

In conclusion, the study suggests that medical therapy combination with BPA is more effective than medical therapy alone. From this we can infer that the patients who do not normalize pulmonary hemodynamics with medical therapy should be offered BPA if appropriate, even though long-term medication.

\section{Abbreviations}


6MWD - 6 minutes walking distance

BPA-Balloon pulmonary angioplasty

$\mathrm{Cl}$ - cardiac index

CO- cardiac output

CTEPH-Chronic thromboembolic pulmonary hypertension

FC- functional class

IVC- inferior vena cava

LV- left ventricular

LVEDD- left ventricular end-diastolic diameter

MPAD- main pulmonary artery diameter

mPAP- mean pulmonary artery pressure

NT-proBNP- N-terminal fragment of pro-brain natriuretic peptide

PEA- pulmonary endarterectomy

$\mathrm{PH}$ - pulmonary hypertension

PVR- pulmonary vascular resistance

RA- right atrial

RV- right ventricular

RV FAC- right ventricular fractional area change

TAPSE- tricuspid annular plane systolic excursion

TRPD- tricuspid regurgitation pressure difference

WHO- World Health Organization

\section{Declarations}

Ethic approval and consent participate 
The study was approved by the ethics committee of Beijing Chaoyang Hospital, Capital Medical University (NO.2019-KE-377).

\section{Consent for publication}

All the patients provided their written informed consents.

\section{Availability of data and materials}

Patient clinical data, RHC and BPA procedures as well as echocardiographic data are fully available in the original format for data transparency. The datasets generated and/or analysed during the current study are available from the corresponding author on reasonable request.

\section{Competing interests}

The authors declare that there have no competing interests.

\section{Funding}

This study is supported by National Natural Science Foundation of China (Grant Nos. 31670928). The funding body had no role in the design of the study, collection, analysis, interpretation of data and in writing the manuscript.

\section{Author contributions}

Y. Yang is the guarantor of this article. W. Wang and S. Yang take responsibility for the integrity of the data and the accuracy of the data analysis. Y. Yang contributed to conception and design, and approved the final manuscript. Q. Huang and J. Wang contributed to the BPA procedures. Y. Li contributed to her assistance with echocardiographic figures. Y. Yang and W. Wang contributed to the statistical analysis and manuscript preparation. J. Gong and T. Kuang contributed to conduct the study and follow-up of patients. All authors have read and approved the manuscript.

\section{Acknowledgments}

Not applicable.

\section{References}

1. Lang IM, Pesavento R, Bonderman D, Yuan JX. Risk factors and basic mechanisms of chronic thromboembolic pulmonary hypertension: a current understanding. Eur Respir J. 2013;41(2):462-68.

2. Galie N, Humbert M, Vachiery JL, Gibbs S, Lang I, Torbicki A, et al. 2015 ESC/ERS Guidelines for the diagnosis and treatment of pulmonary hypertension: The Joint Task Force for the Diagnosis and Treatment of Pulmonary Hypertension of the European Society of Cardiology (ESC) and the European Respiratory Society (ERS): Endorsed by: Association for European Paediatric and 
Congenital Cardiology (AEPC). International Society for Heart and Lung Transplantation (ISHLT); 2015. vol. 46.

3. Jamieson SW, Kapelanski DP, Sakakibara N, Manecke GR, Thistlethwaite PA, Kerr KM, et al. Pulmonary endarterectomy: experience and lessons learned in 1,500 cases. Ann Thorac Surg. 2003;76(5):1457-62, 1462-64.

4. Auger WR, Fedullo PF. Chronic thromboembolic pulmonary hypertension. Semin Respir Crit Care Med. 2009;30(4):471-83.

5. Jais X, D'Armini AM, Jansa P, Torbicki A, Delcroix M, Ghofrani HA, et al. Bosentan for treatment of inoperable chronic thromboembolic pulmonary hypertension: BENEFiT (Bosentan Effects in iNopErable Forms of chronlc Thromboembolic pulmonary hypertension), a randomized, placebocontrolled trial. J Am Coll cardiol. 2008;52(25):2127-34.

6. Suntharalingam J, Treacy CM, Doughty NJ, Goldsmith K, Soon E, Toshner MR, et al. Long-term use of sildenafil in inoperable chronic thromboembolic pulmonary hypertension. Chest. 2008;134(2):22936.

7. Bresser P, Fedullo PF, Auger WR, Channick RN, Robbins IM, Kerr KM, et al. Continuous intravenous epoprostenol for chronic thromboembolic pulmonary hypertension. Eur Respir J. 2004;23(4):595600 .

8. Ghofrani HA, Simonneau G, D'Armini AM, Fedullo P, Howard LS, Jais X, et al. Macitentan for the treatment of inoperable chronic thromboembolic pulmonary hypertension (MERIT-1): results from the multicentre, phase 2, randomised, double-blind, placebo-controlled study. Lancet Respir Med. 2017;5(10):785-94.

9. Simonneau G, Armini D, Ghofrani AM, Grimminger H, Hoeper F, Jansa MM. P et al: Riociguat for the treatment of chronic thromboembolic pulmonary hypertension: a long-term extension study (CHEST2). Eur Respir J. 2015;45(5):1293-302.

10. Ghofrani HA, D'Armini AM, Grimminger F, Hoeper MM, Jansa P, Kim NH, et al. Riociguat for the treatment of chronic thromboembolic pulmonary hypertension. N Engl J Med. 2013;369(4):319-29.

11. Voorburg JA, Cats VM, Buis B, Bruschke AV. Balloon angioplasty in the treatment of pulmonary hypertension caused by pulmonary embolism. Chest. 1988;94(6):1249-53.

12. Mizoguchi $H$, Ogawa A, Munemasa $M$, Mikouchi $H$, Ito $H$, Matsubara $H$. Refined Balloon Pulmonary Angioplasty for Inoperable Patients with Chronic Thromboembolic Pulmonary Hypertension. Circ Cardiovasc Interv. 2012;5(6):748-55.

13. Delcroix M, Torbicki A, Gopalan D, Sitbon O, Klok FA, Lang I, et al: ERS statement on chronic thromboembolic pulmonary hypertension. Eur Respir J. 2021, 57(6).

14. Rubin LJ, Galie N, Grimminger F, Grunig E, Humbert M, Jing ZC, et al. Riociguat for the treatment of pulmonary arterial hypertension: a long-term extension study (PATENT-2). Eur Respir J. 2015;45(5):1303-13.

15. Lewczuk J, Piszko P, Jagas J, Porada A, Wojciak S, Sobkowicz B, et al. Prognostic factors in medically treated patients with chronic pulmonary embolism. Chest. 2001;119(3):818-23. 
16. Marra AM, Halank M, Benjamin N, Bossone E, Cittadini A, Eichstaedt CA, et al. Right ventricular size and function under riociguat in pulmonary arterial hypertension and chronic thromboembolic pulmonary hypertension (the RIVER study). Respir Res. 2018;19(1):258.

17. Brenot P, Jais X, Taniguchi Y, Garcia AC, Gerardin B, Mussot S, et al: French experience of balloon pulmonary angioplasty for chronic thromboembolic pulmonary hypertension. Eur Respir J. 2019, 53(5).

18. Kwon W, Yang JH, Park TK, Chang SA, Jung DS, Cho YS, et al. Impact of Balloon Pulmonary Angioplasty on Hemodynamics and Clinical Outcomes in Patients with Chronic Thromboembolic Pulmonary Hypertension: the Initial Korean Experience. J Korean Med SCI. 2018;33(4):e24.

19. Ogawa A, Satoh T, Fukuda T, Sugimura K, Fukumoto Y, Emoto N, et al: Balloon Pulmonary Angioplasty for Chronic Thromboembolic Pulmonary Hypertension. Circulation: Cardiovascular Quality and Outcomes. 2017, 10(11).

20. Sugimura K, Fukumoto Y, Satoh K, Nochioka K, Miura Y, Aoki T, et al. Percutaneous Transluminal Pulmonary Angioplasty Markedly Improves Pulmonary Hemodynamics and Long-Term Prognosis in Patients with Chronic Thromboembolic Pulmonary Hypertension. CIRC J. 2012;76(2):485-88.

21. Wiedenroth CB, Ghofrani HA, Adameit MSD, Breithecker A, Haas M, Kriechbaum S, et al. Sequential treatment with riociguat and balloon pulmonary angioplasty for patients with inoperable chronic thromboembolic pulmonary hypertension. Pulm Circ. 2018;8(3):767763716.

22. Broch K, Murbraech K, Ragnarsson A, Gude E, Andersen R, Fiane AE, et al. Echocardiographic evidence of right ventricular functional improvement after balloon pulmonary angioplasty in chronic thromboembolic pulmonary hypertension. J Heart Lung Transplant. 2016;35(1):80-6.

23. Shimokawahara $H$, Ogawa A, Mizoguchi H, Yagi H, Ikemiyagi $H$, Matsubara $H$ : Vessel Stretching Is a Cause of Lumen Enlargement Immediately After Balloon Pulmonary Angioplasty. Circulation: Cardiovascular Interventions. 2018, 11(4).

24. Hosokawa K, Abe K, Horimoto K, Yamasaki Y, Nagao M, Tsutsui H. Balloon pulmonary angioplasty relieves haemodynamic stress towards untreated-side pulmonary vasculature and improves its resistance in patients with chronic thromboembolic pulmonary hypertension. Eurointervention. 2018;13(17):2069-76.

25. Magon W, Stepniewski J, Waligora M, Jonas K, Podolec P, Kopec G. Pulmonary Artery Elastic Properties After Balloon Pulmonary Angioplasty in Patients with Inoperable Chronic Thromboembolic Pulmonary Hypertension. Can J Cardiol. 2019;35(4):422-29.

\section{Figures}



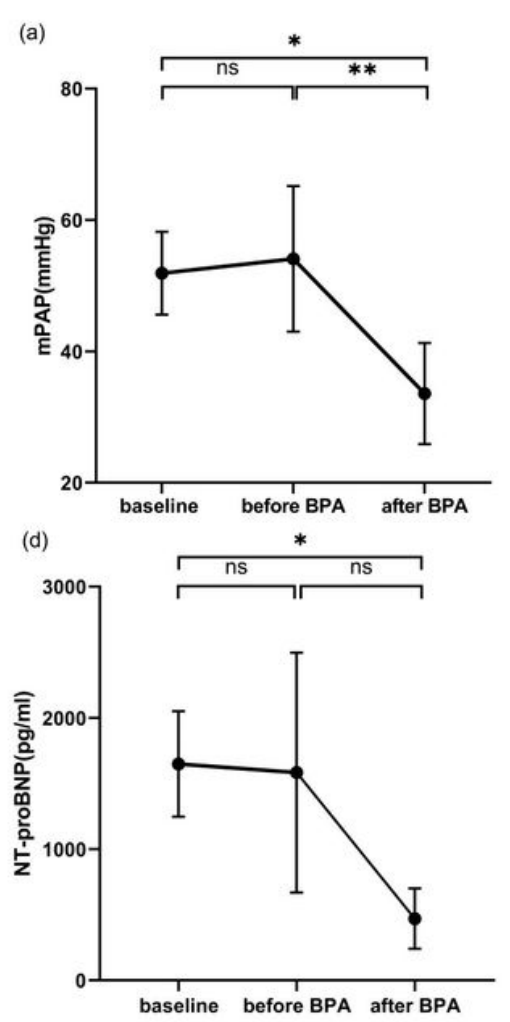
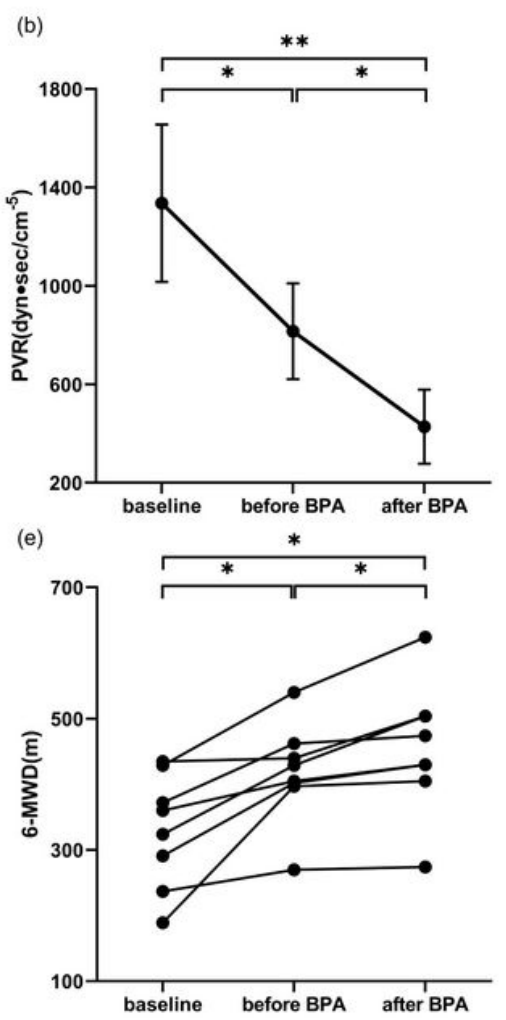
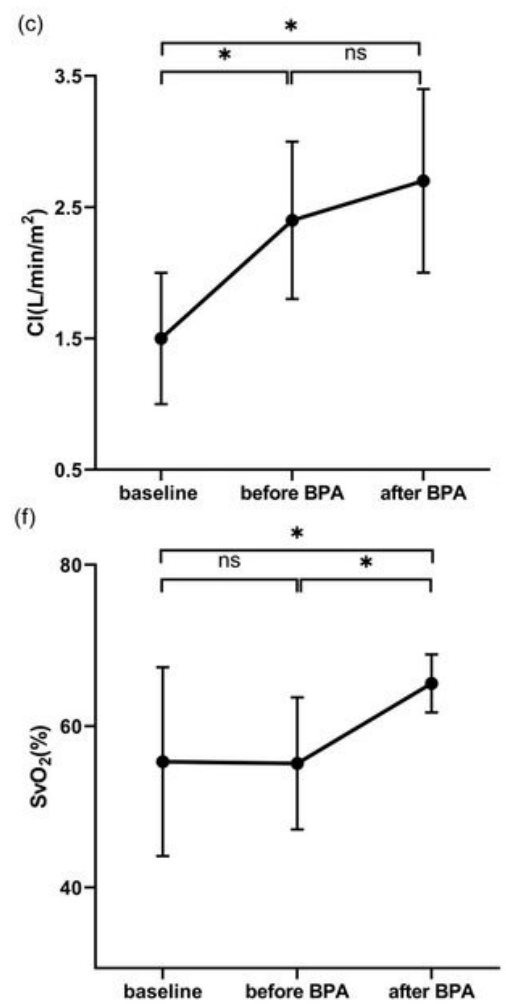

Figure 1. Effects of riociguat and combination BPA on hemodynamics and exercise capacity. (a), mPAP; (b), PVR; (c), CI; (d), NT-proBNP; (e), 6MWD; (f), $\mathrm{SvO}_{2}$. ns indicates no significance, ${ }^{*} \mathrm{P}<0.05,{ }^{*} * \mathrm{P}<0.01 .6 \mathrm{MWD}=6$-minute walk distance; $\mathrm{BPA}=$ balloon pulmonary angioplasty; $\mathrm{CI}=$ cardiac index; $\mathrm{mPAP}=$ mean pulmonary artery pressure; $\mathrm{NT}$-proBNP= $\mathrm{N}$-terminal pro-brain natriuretic peptide; $\mathrm{PVR}=$ pulmonary vascular resistance; $\mathrm{SvO}_{2}=$ mixed venous oxygen saturation.

\section{Figure 1}

Effects of riociguat and combination BPA on hemodynamics and exercise capacity. (a), mPAP; (b), PVR; (c), $\mathrm{Cl}$; (d), NT-proBNP; (e), 6MWD; (f), $\mathrm{SvO}_{2}$. ns indicates no significance, ${ }^{\star} \mathrm{P}<0.05$, ${ }^{\star \star} \mathrm{P}<0.01 .6 \mathrm{MWD}=6$ minute walk distance; $\mathrm{BPA}=$ balloon pulmonary angioplasty; $\mathrm{Cl}=$ cardiac index; $\mathrm{mPAP}=$ mean pulmonary artery pressure; $\mathrm{NT}$-proBNP= N-terminal pro-brain natriuretic peptide; $\mathrm{PVR}=$ pulmonary vascular resistance; $\mathrm{SvO}_{2}=$ mixed venous oxygen saturation. 


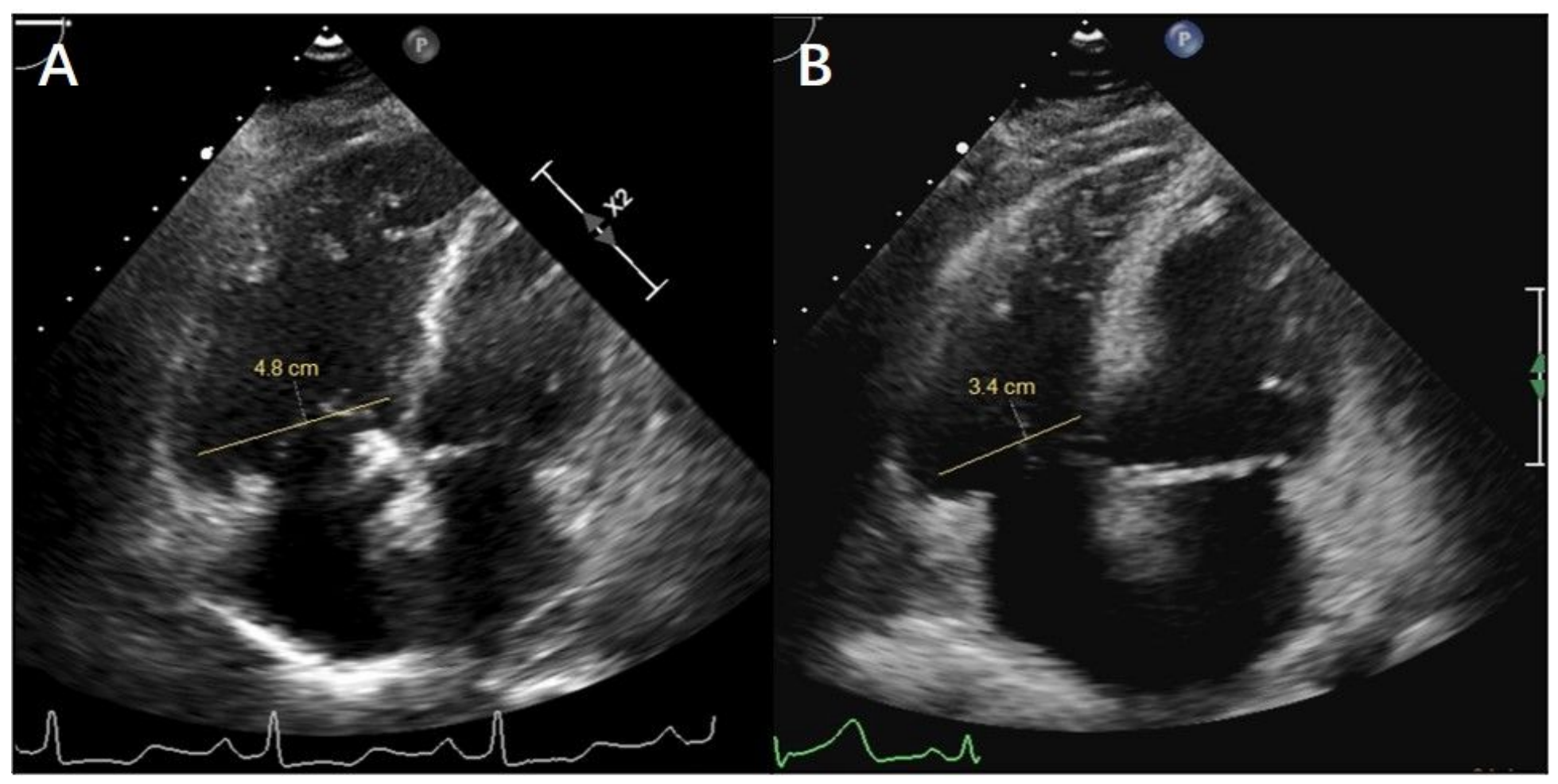

Figure 2. RV basal diameter. Measurements of RV size were made in dedicated RV views. This image illustrates measurements of RV diameter at the time of before BPA(A) and follow-up after treatment with $\mathrm{BPA}(\mathbf{B})$. $\mathrm{BPA}=$ balloon pulmonary angioplasty; $\mathrm{RV}=$ right ventricular.

\section{Figure 2}

$\mathrm{RV}$ basal diameter. Measurements of RV size were made in dedicated RV views. This image illustrates measurements of RV diameter at the time of before BPA(A) and follow-up after treatment with BPA(B). $\mathrm{BPA}=$ balloon pulmonary angioplasty; $\mathrm{RV}=$ right ventricular. 


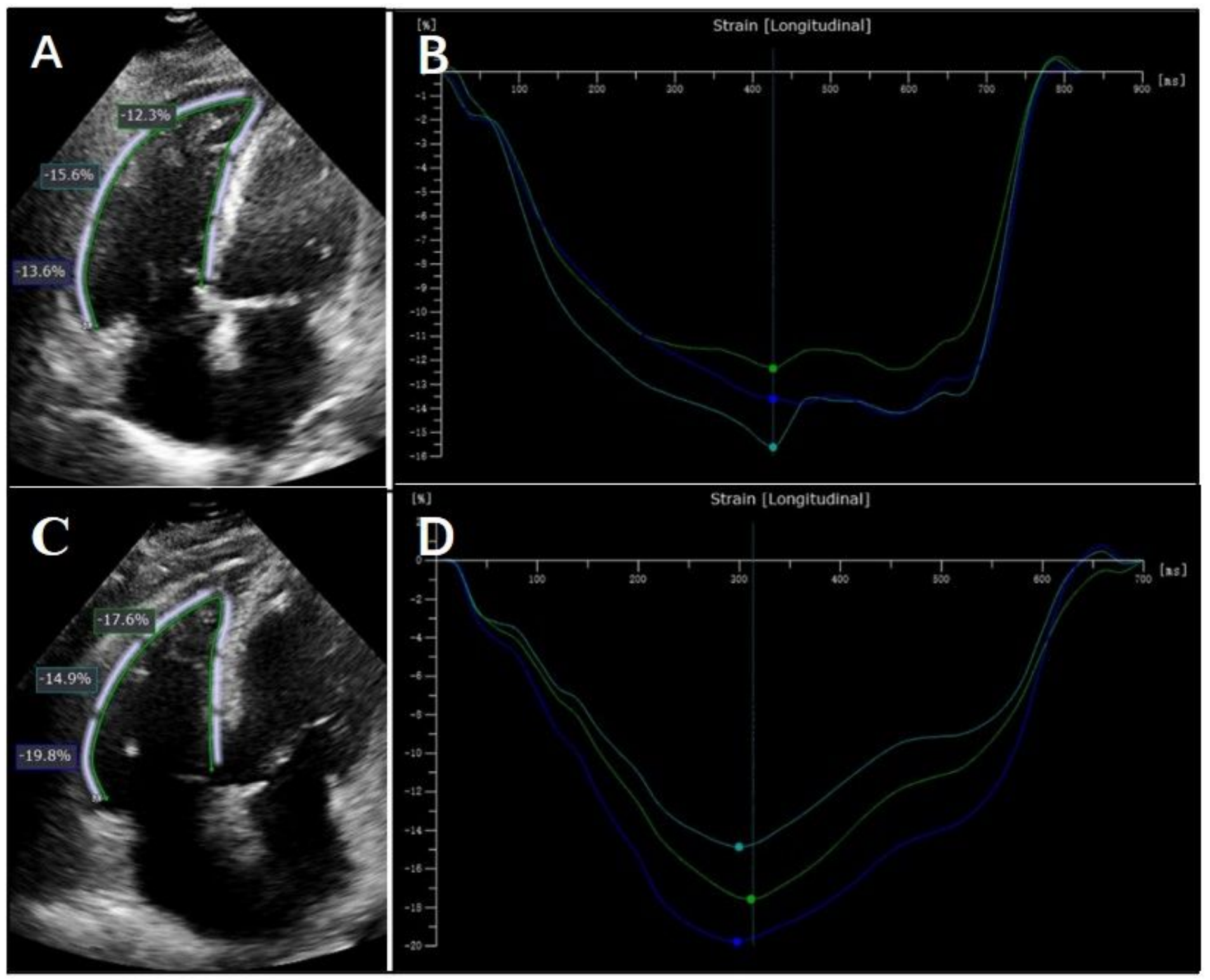

Figure 3. Analysis of RV longitudinal strain using speckle-tracking imaging. RV strain from the same representative patient before (A and $\mathbf{B})$ and after $(\mathbf{C}$ and $\mathbf{D})$ treatment with BPA. Before treatment, there is severe RV dysfunction. After BPA, systolic function is improved in base and apex segments of RV free wall (green and blue traces). BPA= balloon pulmonary angioplasty; $\mathrm{RV}=$ right ventricular.

\section{Figure 3}

Analysis of RV longitudinal strain using speckle-tracking imaging. RV strain from the same representative patient before (upper panel) and after (lower panel) treatment with BPA. Before treatment, there is severe $R V$ dysfunction. After BPA, systolic function is improved in base and apex segments of RV free wall (green and blue traces). BPA= balloon pulmonary angioplasty; $\mathrm{RV}=$ right ventricular. 